\title{
Computational algorithms for extraction and analysis of two-dimensional transportation networks
}

\author{
Werner Baumgarten and Marcus J.B. Hauser \\ Manuscript received on January 10, 2012 / accepted on September 21, 2012
}

\begin{abstract}
Transportation networks are of paramount importance in natural and man-made systems. We present algorithms to extract twodimensional real world networks from images, thus making the networks available for in silico analysis. The algorithms deal with image processing, network extraction, and correction of reconstruction errors. Next, methods are presented to obtain the geometrical and topological structure of the network. This yields the graph underlying the network, which can be subjected to graph theoretical analysis. The statistical distributions of the lengths, widths, and areas of the vein segments, as well as the angles between veins departing from a branching point are investigated. In a case study, the algorithms are applied to the tubular vein network of the myxomycete Physarum polycephalum. Despite of the large variability between different network realizations, statistics obtained from cummulated experiments correspond to those extracted from individual experiments.
\end{abstract}

Keywords: Real network, Physarum polycepha/um, venation, data extraction.

\section{INTRODUCTION}

In today's world, networks, in all of their variations, are of immense significance as, for instance, social [1], economic [2], or transportation networks. The latter include air-transportation $[3,4]$, railway [5] and urban railway networks [6], electric powergrids [7] as well as ant cave networks $[8,9,10]$. There are also living transportation networks, like the blood vascularization system [11], leaf vessel networks [12,13], and the tubular network of Physarum polycephalum $[14,15]$.

In the present paper we analyze the biological transportation network of Physarum polycephalum, a slime mould with some remarkable idiosyncrasies. Experiments are performed using the plasmodial state of $P$. polycepha/um, which consists of a very dense apical front domain that is connected to a two-dimensional vein network, in which protoplasm is periodically transported back and forth by peristaltic pumping (Fig. 1). This plasmodium that is formed by a single cell evolves in time and its size may range from $10 \mu \mathrm{m}^{2}$ to $1 \mathrm{~m}^{2}$.

The plasmodium of $P$. polycepha/um forms regular graphs with the unique node degree $k=3$ [15]. The network is dynamic and it adapts to external, environmental conditions. During adaptation, it has been shown that the network optimizes itself with respect to at least three factors, namely transport efficiency, cost minimization, and error tolerance [16]. These intriguing characteristics allow P. polycephalum to solve graph theoretical problems, like the construction of Steiner trees or minimal pathways $[17,18]$, or even to mimic the railway network in the vicinity of Tokyo $[16,19]$. Such interesting networks existing in the real world call for a more detailed study of their characteristics and

Correspondence to: Marcus J.B. Hauser - E-mail: marcus.hauser@ovgu.de - http://www.biophysik.ovgu.de Abteilung Biophysik, Institut für Experimentelle Physik, Otto-von-Guericke-Universität Magdeburg, 39106 Magdeburg, Germany. E-mail: werner.baumgarten@gmail.com 


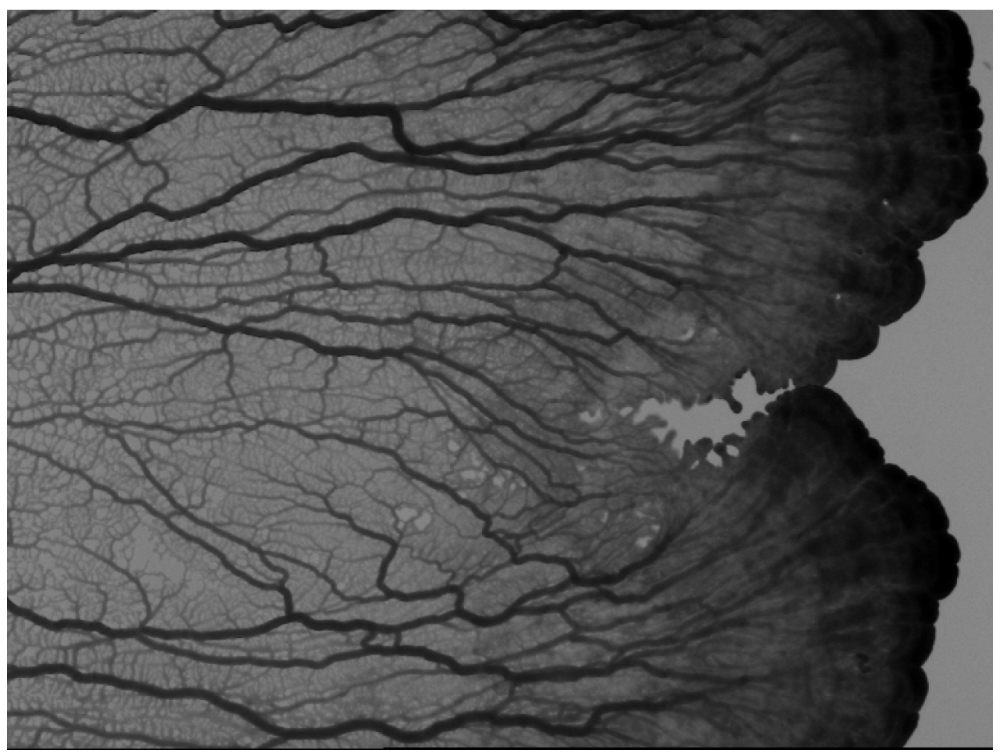

Figure 1 - Plasmodium of the slime mould Physarum polycephalum. The dense apical front zone (right) is connected to a network of veins (left). Image size: $3.6 \mathrm{~cm} \times 2.5 \mathrm{~cm}$.

properties. To this purpose, the real networks need to be detected and transformed into a digital network, such that the network is accessible for in silico algorithmic analysis.

The purpose of this paper is fourfold. First, we wish to present algorithms and techniques to extract and reconstruct two-dimensional networks from images, thus making them available for computer-based analysis. Second, we present a series of algorithms for the geometrical and topological analysis of the networks. Third, we apply the algorithms to a case study, namely the tubular vein network of $P$. polycephalum. Last but not least, we also show that the resulting network statistics of cumulative and individual experiments yield similar results.

\section{EXPERIMENTAL METHODS}

\subsection{Sample preparation and monitoring}

Experiments were started by placing a small rectangular filter paper $\left(1 \times 6 \mathrm{~cm}^{2}\right)$ with sclerotia of Physarum polycepha/um strain HU195 $\times$ HU200 on a $1 \%(w / v)$ agar gel close to the edge of a covered $10 \times 10 \mathrm{~cm}^{2}$ Petri dish. The sclerotium is a dormant, dehydrated form of $P$. polycephalum, which can transform back into the plasmodial form when the environmental conditions become favourable again.

The Petri dish was placed on a heat absorbing water bath, and illuminated by a cold light source (Polytec) emitting white light from below while being monitored by a CCD camera
(Hamamatsu C3077) from above. Images were collected with a resolution of $768 \times 576$ pixels corresponding to an area of $3.5 \times 2.6 \mathrm{~cm}^{2}$ with a resolution of $0.0456 \mathrm{~mm} /$ pixel. For temporal evolution experiments, images were collected every $30 \mathrm{~s}$. All images are grey-scaled and transferred to a computer for subsequent analysis with self-written algorithms. We concentrated on the tubular vein network behind the apical zone to obtain the statistics of the important network parameters (Figs. 1 and 2). The relevant data was extracted from images (movies) of the network, using the algorithms presented in section 3 .

\subsection{Statistics}

Several characteristic features of the transportation networks can be captured by statistics, e.g., the distributions of lengths, widths, and areas of the veins forming a network.

We adopt two strategies to obtain statistical data. The first consists in obtaining statistical data from a single experiment. Such 'individual statistics' work well, as long as the number of veins in the frame is sufficiently high. The second approach is based on the accumulation and extraction of data obtained under similar conditions from a large number of experiments.

In the present article, we wish to investigate if the statistical information obtained from individual experiments corresponds to that extracted from cumulative data, or if the biological variability in our samples is too pronounced to allow for cumulative statistics. 


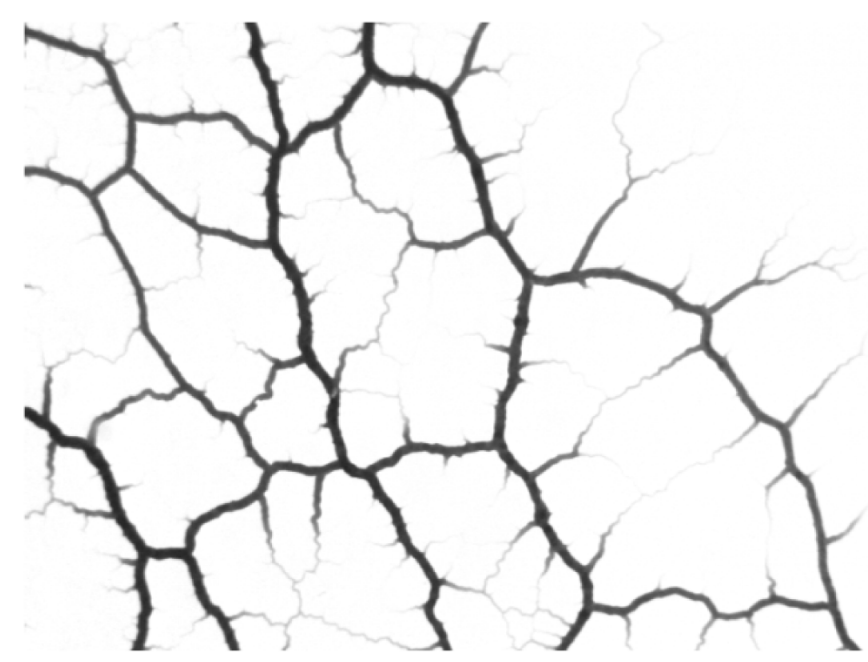

Figure 2 - Background subtracted image of a part of a vein network formed by the plasmodium of the slime mould Physarum polycepha/um, $16 \mathrm{~h}$ after placing sclerotia on the Petri dish. Image size: $3.6 \mathrm{~cm} \times 2.5 \mathrm{~cm}$.

\subsubsection{Individual Statistics}

Network structures of Physarum polycephalum were analyzed. The distributions of lengths and widths of veins, as well as the distribution of angles at branching points of veins were extracted from single images of the plasmodial network. The temporal evolution of a network was always analyzed using consecutive individual images of a movie.

\subsubsection{Cumulative Statistics}

A total of 121 independent networks were analyzed exactly $24 \mathrm{~h}$ after placing the scelerotia on the agar, i.e. after starting the experiment. The data on the lengths, widths, and areas of the veins, as well as the angles between the veins were extracted from all 121 images, and collapsed into the same statistics. This procedure provides a huge number of events, leading to a much smoother statistical distribution, as compared to the data collected from individual images.

However, at the instant when we collected data for the cumulative statistics (as well as for any other instant during the experiments), there was a considerable biological variability with respect to the individual networks, especially concerning the number of veins formed. Therefore, it is important to investigate whether the statistical distributions retain their functional form in the individual and the cumulative statistics.

\section{IMAGE PROCESSING}

To detect the properties of a network formed by the plasmodium of Physarum polycepha/um the individual images of the movie were subjected to image processing. The network in Figure 2 forms a planar, weighted, undirected graph, with the veins as edges and the branching points of veins as nodes. Hence, the graph matrix $G$ can be written as:

$$
G_{i j}=F_{i j} \cdot A_{i j}=W_{i j} \cdot L_{i j} \cdot A_{i j},
$$

where $A$ is the adjacency matrix, containing the information whether node $i$ is connected to node $j$. If this is the case, the entries of $A_{i j}$ and $A_{j i}$ will be 1 , else 0 . The weighting matrix $F$ contains information about the area of veins as well as on the width $W_{i j}$ and length $L_{i j}$ of any edge $i j$ connecting nodes $i$ and $j$. Therefore, the image processing algorithms must measure the length, width, and area of each vein in the network as accurately as possible. These information should be stored in different matrices for later analysis.

In the following, we will describe the image processing steps and the analysis of the network. The individual steps are summarized as a flow chart in Figure 3.

First, the network must be extracted from the recorded image. In studies of the temporal evolution any stationary inhomogeneities are eliminated by background subtraction. On the other hand, when only snapshots of the network are analyzed, i.e. when the temporal evolution of the spatial domain is not explicitly analyzed, the background is not subtracted. The resulting image is binarized using an appropriate threshold estimated by the experimenter. The threshold should remain the same within a time series, otherwise the illumination conditions are no longer constant, leading to incomparable results. The process of binarization may create spurious gaps (i.e. non-recognized pixels) in the network. 


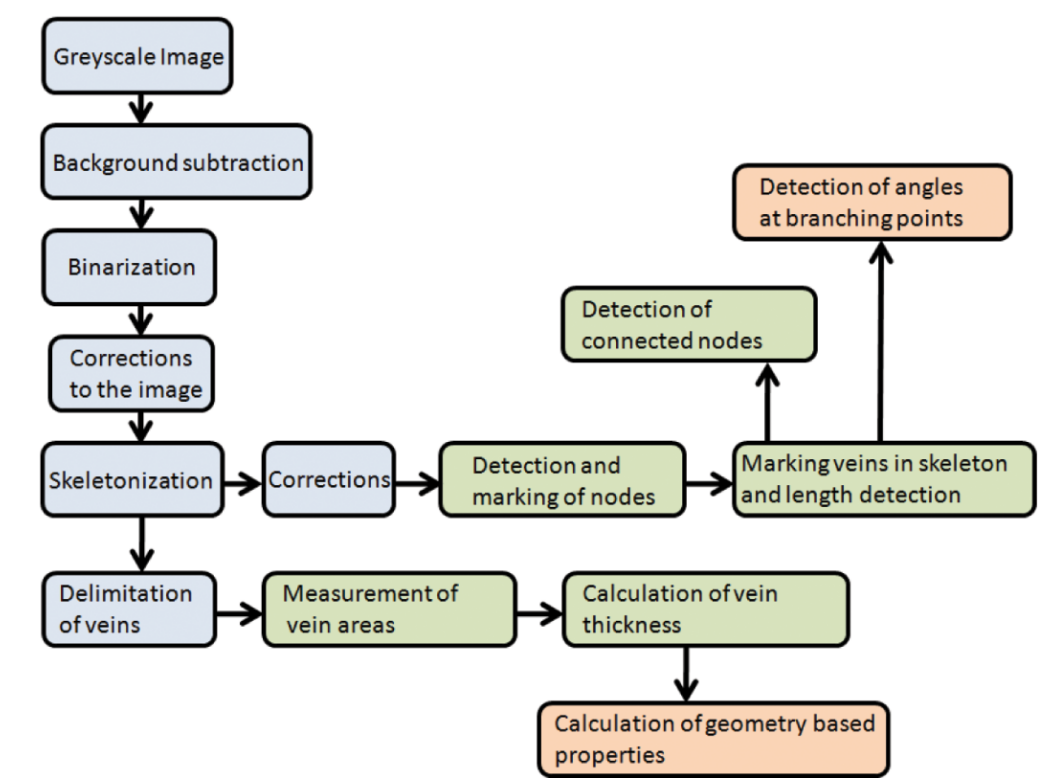

Figure 3 - Flow chart illustrating the steps to extract networks from images. Image processing steps are compiled in blue boxes, while steps to obtain the network statistics are labeled in green. Red boxes represent other steps.

A series of algorithms was applied to correct for these binarization errors [20].

\subsection{Corrections of the binarized image}

Algorithms to correct for spurious gaps of one or two pixels size were described in ref. [20]. These corrections lead to a smoothing of the veins, thus increasing the rate of successful detections and extractions of the veins from the images. However, using these corrections in a very dense network leads to overcorrections, such that veins in an immediate vicinity may become connected, even though they were not in the original image. Therefore, these corrections should only be applied when required.

\subsection{Detection of nodes and veins in the skeletonized network}

In a next step, the corrected binarized network is skeletonized. That means that every network segment in the image is collapsed onto an one-pixel thin line using the skeletonization algorithm proposed by Zhang and Suen [21].

Since our algorithms scan the matrix of pixels exclusively along horizontal and vertical directions, i.e., along the lines and columns of pixels, all veins need to be tracked along these directions. Obviously, this may lead to problems when the vein extends diagonally. Thus, so called diagonal corrections must be applied [20]. They consist in adding pixels to the diagonal vein, such that they form 'stairs', thus allowing for a tracking of veins by exclusively following the object in horizontal and vertical directions. By adding such stairs, nodes and vein segments are detectable within the skeletonized network.

The position of the nodes and veins have to be extracted from the skeletonized network and these information should be stored. It is useful to work with an image matrix $I$, which has the size of the image measured in pixels. Each entry of $I$ will be labelled either as a node, as belonging to a vein or edge, or as background.

When searching for nodes, we assume that the nodes in the skeletonized picture are located at the same positions as in the binarized picture. In skeletonized networks, different types of branching points (nodes) must be considered. They differ with respect to the number of pixels that form the node, as shown in Figure 4.

Nodes consist either of 4 pixels or of only 1 pixel. For the subsequent analysis of the network, it is important to work only with nodes consisting of a single pixel, therefore those consisting of 4 pixels must lose one pixel before nodes are detected, such that the skeletonized version of the node is just a single pixel. That means that before the nodes are detected, a mask should be swept over the entire picture, searching the image matrix for nodes made of 4 pixels. These nodes should then be simplified to nodes of a single pixel.

During the detection of nodes it is also important to enumerate nodes, in order to identify them, and to save them in the image 


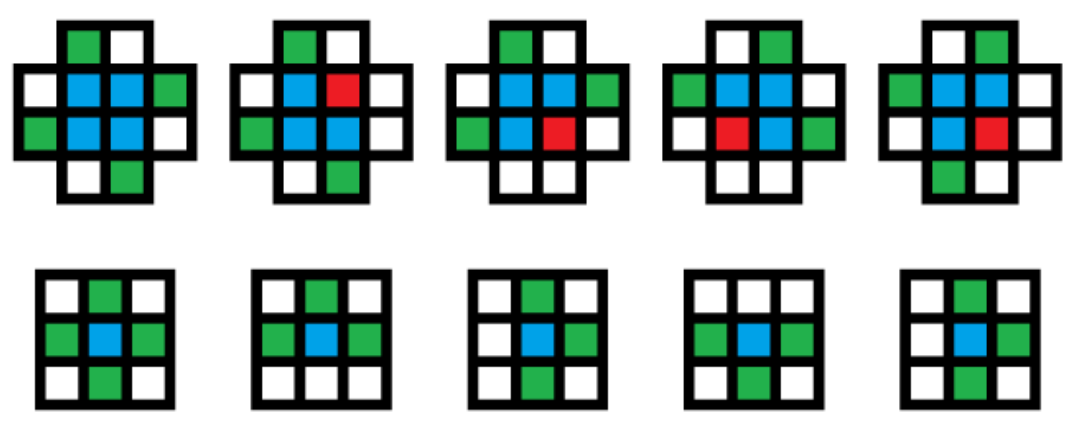

Figure 4 - Node types that can occur at branching points of veins in a skeletonized network. White pixels represent the background, while blue and green pixels represent nodes and veins, respectively. Red pixels are the pixels that should be deleted. Note that not all symmetric cases are shown.

matrix $I$. Knowing the positions of all nodes in the network, we scan through the entire image, and whenever a node is found, we follow the connected veins to their ends, be it either another node, or an open end (meaning that the venule ends in the background). If we find two nodes $i$ and $j$ to be connected, this is saved at the entries $A_{i j}$ and $A_{j i}$ of the adjacency matrix $A$.

While tracking each vein starting from a node, its length $l$ is measured by counting the number $p$ of pixels that make up the vein in the skeletonized image. The length $l$ is determined precisely by subtracting $s$ from $p$, where $s$ is the number of staircorrections applied to this vein according to:

$$
l=p-s(3-\sqrt{2}) .
$$

This is done to correct the length of veins containing diagonal elements. At this point we save the length of the vein in the corresponding length matrix $L$.

\subsection{Angles at branching points}

The angles at branching points are calculated directly from the corrected skeletonized network. The rationale of detecting the angles consists in tracking each vein leaving a node up to a certain pixel and store the coordinate of that last pixel. The 3 or 4 coordinates obtained by that recipe determine a triangle or rectangle with the node in its centre. The angles are measured by trigonometry in these geometric objects.

To determine the angles at branching points, we navigate to a node of choice. We store the geometric form of the vein into an array by mapping its geometry to a symbolic sequence, where the set of numbers $\{1,2,3,4\}$ identifies the directions \{up, right, down, left\}.

For example, let a vein leave the node in upward direction. Thus, the first pixel forming this vein is located 'above' the node, therefore, we write a '1' into the first position of the array. We now step on this pixel and examine its 8-pixel-neighbourhood to find the next pixel of the vein. Let the next pixel of the vein be oriented to the right of the current one, then we note a ' 2 ' at position 2 and so on. An illustration of the mapping is given in Figure 5. The processes of coding and storing the structure of the vein leaving a node is repeated for all veins leaving a node.

The accuracy of the detection of angles depends on the number of pixels that are tracked along the veins emanating form a node. The longer the tracked distance along the vein, the more accurate the angles can be determined. The resolution of the image also matters: a higher resolution demands a larger numbers of pixels to be tracked, but is more accurate. For our resolution $(768 \times 576$ px $) 15$ pixels were used to track the vein.

After tracking all veins departing from a node, the node is taken as the origin of a coordinate system and angles are calculated by trigonometry and stored for later analysis. Figure 6 illustrates the geometry of the problem. For our experiments we have found the largest error to be $\pm 11^{\circ}$.

\subsection{Determination of the borders between vein segments}

The next task is to determine the borders between vein segments in the binarized (and corrected) network. To this purpose, the following steps are repeated for each node of the skeletonized network (Fig. 7).

Borders between vein segments need to be created. To this purpose a circle, with the node as centre and the radius $r$, is drawn around the neighbourhood of every node. For a properly chosen radius $r$ this step leads to three distinct background areas. In each of them the background pixel that has the shortest distance to the node point is marked and directly connected to the node. 


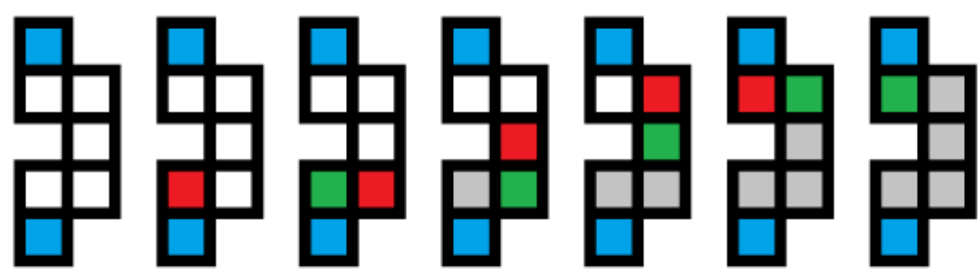

Figure 5 - Process of tracking a vein. The green pixel marks the current pixel. Pixels labelled in blue represent nodes, white pixels are (yet) unvisited and grey ones are visited pixels of the vein. Red pixels are the next targeted pixels. Here, the starting point for tracking the veins structure is the bottom blue node. The representation of this vein in symbolic sequence is $\{1,2,1,1,4\}$.
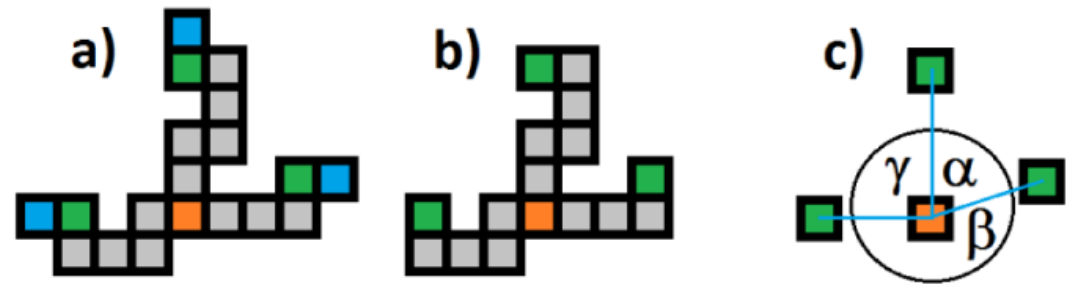

Figure $\mathbf{6}$ - Determination of the angles between veins. a) The pixel marked in orange is the starting node. Three veins leave from it. b) Their end points are marked in green. c) Direct connections from the origin to the end points are drawn. The angles $\alpha, \beta$ and $\gamma$ determined by these connections are calculated.
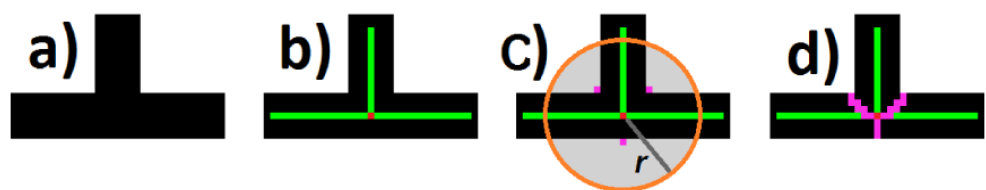

Figure 7 - The steps to create borders between veins. a) Original node. b) The skeletonized image is overlayed onto the original image. The node is marked in red, the veins in green. c) Circle around the node, dividing the background into three distinct areas. The background pixels with the shortest connection to the node point in the skeleton are marked in purple. d) Direct connections between the nearest background pixels and the node point are drawn (purple lines). These connections represent the borders of the veins.

These borders between nodes are stored within the image matrix $I$. Delimiting veins in this way will sometimes lead to errors. They occur more often in very dense networks. For our setup we obtained about $2 \%$ of erroneous delimitations. Figure 8 shows two examples to which the algorithms were applied.

\subsection{Determination of areas and thicknesses of veins}

The area of a vein segment is determined by counting the pixels that are found between the borders of two connected nodes. By dividing the area through the length of the vein, a mean thickness is calculated, which corresponds to - if we assume cylinders as the shape of veins - the diameter of these cylindrical tubes. The widths are saved in the width matrix $W$.

\section{CASE STUDY: THE PLASMODIAL VEIN NETWORK OF Physarum polycephalum}

In this section we apply the data imaging and network analysis algorithms introduced in section 3 to study the plasmodium of the slime mould $P$. polycephalum, which forms a two-dimensional tubular vein network. Furthermore, we compare the statistical results obtained from a single image with statistics obtained from cumulative data (as described in section 2.2). This comparison will shed light on the question, whether the accumulation of images still preserves the statistics found for individual images.

\subsection{Distribution of lengths of vein segments}

The distribution of lengths of vein segments follows a log-normal distribution when one considers the entire spectrum of lengths 

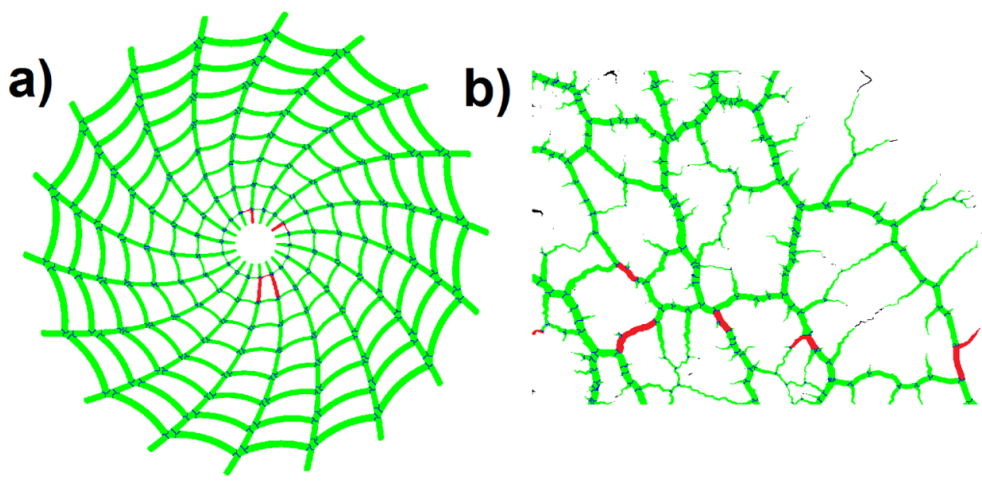

Figure 8 - a) Schematic representation of a cobweb consisting of 445 veins and 285 nodes. The green areas had been successfully delimited, while red ones have not. During the delimitation of threads 4 errors occurred, which give a $1.4 \%$ error fraction. The mean thickness of threads is 6.9 px. b) A Physarum polycepha/um network consisting of 479 veins and 255 nodes. Veins marked in green are successfully delimited, red ones are not. Here 6 errors occurred, yielding an error fraction of $1.6 \%$. The mean thickness of the veins is $4.6 \mathrm{px}$.

(Fig. 9). The error in the distribution is larger for smaller lengths than for longer ones. This is due to the resolution of the image, which causes short veins not to be recognized accurately enough. It is important to note that both, the cumulative experiments (summing over 121 images, yielding 27000 veins) and the individual experiment (with roughly 500 veins) show the same type of normalized log-normal distribution.

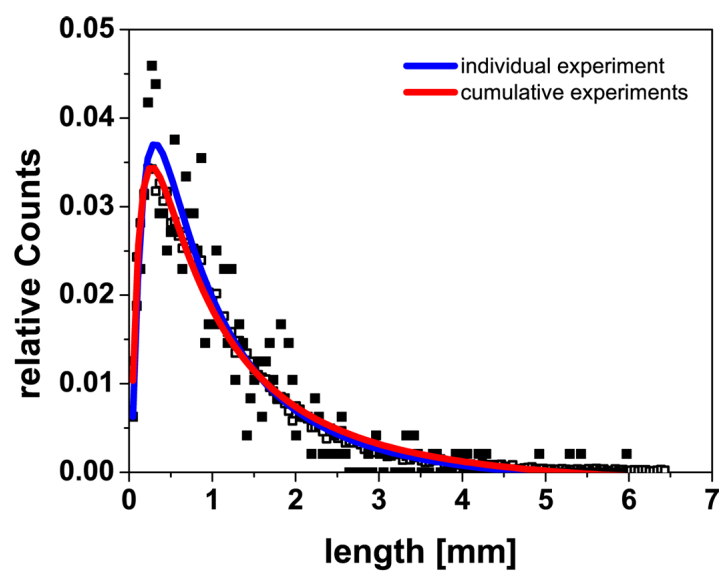

Figure 9 - Log-normal distribution of lengths of vein segments, after accumulation of experiments (red line, full squares), and in an individual experiment (blue line, open squares).

From the lengths $l_{i}$ of all $N$ veins in the skeletonized network the mean length

$$
<l>=\frac{1}{N} \sum_{i=1}^{N} l_{i}
$$

of the network was computed. It increases linearly with time (Fig. 10a). The increase of $<l>$ is caused by the continuous removal of veins from the network, due to the coarsening of the network. The number of veins $N$ in the network decreases with time (Fig. 10b). This process is exponential with a decay constant $\lambda$ :

$$
N(t)=N_{0} e^{-\lambda t} .
$$

From individual experiments the decay constant was found to be $\lambda=(1.4 \pm 0.9) \cdot 10^{-4} s^{-1}$.

a)

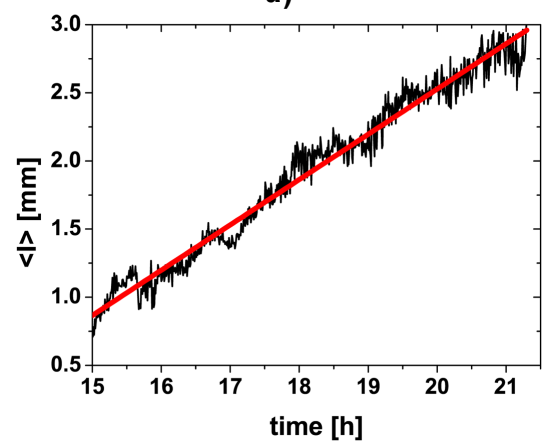

b)

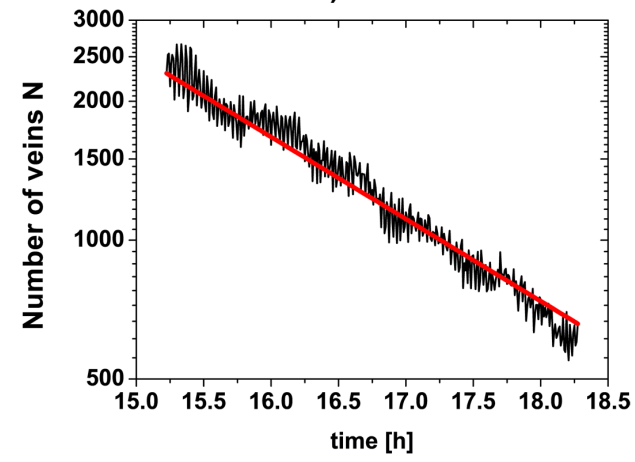

Figure 10 - a) Temporal development of the mean length of the veins, and b) of the number of veins in the network of $P$. polycepha/um. Data obtained from an individual experiment. 


\subsection{Distribution of vein thicknesses}

We found that vein thicknesses are distributed log-normally [15], a finding that has been confirmed by lto et al. [22] recently. In the data presented here, the resolution is $0.04 \mathrm{~mm} / \mathrm{px}$, which is slightly higher than the $0.065 \mathrm{~mm} / \mathrm{px}$ by lto et al. [22], and the $0.08 \mathrm{~mm} / \mathrm{px}$ from our previous study [15]. Using the method presented in section 3.4 we estimate the thicknesses of the veins. Figure 11 shows the statistics of vein thickness for cumulative experiments and for an individual experiment. Both distributions are of the same type, i.e., log-normal.

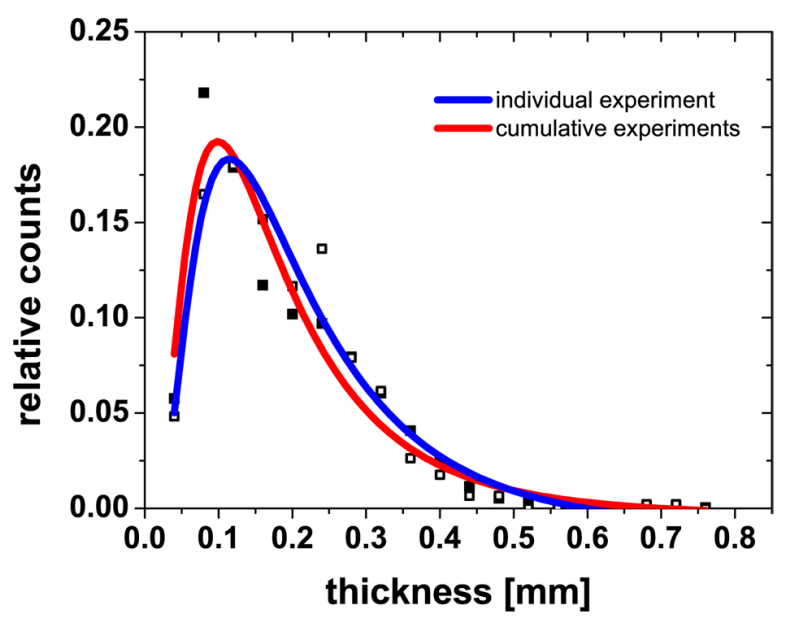

Figure 11 - Distribution of the thicknesses of 27000 investigated veins (cumulative experiments, blue line, full squares) and roughly 500 veins (single experiment, red line, open squares).

\subsection{Distribution of angles}

The distribution of angles at branching points of veins in the skeletonized network is investigated. The result of the cumulative statistics of 121 network images shows a broad and unspecific peak between $90^{\circ}$ and $150^{\circ}$ (Fig. 12a). A second, narrower and more pronounced maximum is found at $180^{\circ}$. The angular distribution depends slightly on the relative widths $w_{1}, w_{2}, w_{3}$ of the veins at a node, where $w_{1} \leq w_{2} \leq w_{3}$. When the node connects a narrow venule to a thick vein, i.e. for $0.2 \leq \frac{w_{1}}{w_{3}} \leq$ 0.3 , angles around $90^{\circ}$ and $180^{\circ}$ are more abundant than for the branches of similar thickness ( $\left.0.7 \leq \frac{w_{1}}{w_{3}} \leq 0.95\right)$ (Fig. 12b).

With time, changes of the angles at a branching point were not observed, i.e. the angles remain fixed once formed.

\section{DISCUSSION}

This article reports on algorithms and analysis tools to investigate real two-dimensional networks originally obtained from images, followed by a case study using the tubular vein network of the myxomycete Physarum polycephalum. We have introduced a step-by-step programme to extract the network and analyze some of its characteristic features, like the lengths, areas, and thicknesses of the veins in networks, as well as the angles at which they leave from the branching points. The algorithms are very flexible such that they can be employed to determine and analyze a wide variety of two-dimensional networks found in the real world. Possible applications could involve the detection and analysis of cobwebs, trail and road networks, and many more. It is worth to note that related algorithms have also been developed for the analysis of leaf veneation networks [12].

The position of the nodes points and the lengths of edges in the network are determined from the skeletonized image, while the areas and thicknesses are found by combining the skeletonized network and the binarized, corrected image. While the determination of lengths works error-free, setting the limits of vein segments is a procedure that may lead to errors. The delimitation of vein segments is performed using data form the binarized, corrected images, and the problem becomes more pronounced as the vein thickness increases. This is due to the skeletonization which is not invariant with respect to the position of nodes. Specially for thick veins, the position of nodes may be shifted slightly from the centre axis of a vein. In other words, the method of delimiting edges works best in networks that are not too dense and which do not possess too thick veins. In our case study on the tubular network of Physarum polycephalum we estimate that the erroneous assessment of vein thicknesses is $\sim 2 \%$.

We introduced an algorithm to determine the angles between veins leaving from nodes. Here, too, the resolution of the primary image matters: the higher the resolution, the lower is the error in the determination of angles; in contrast, at high resolutions only a small number of angles may be determined. Another factor affecting the quality of the angle detection is the number of pixels of a vein emanating from a node which are followed for the angle detection. In principle, a tracking over a larger number of pixels reduces the errors in angle assessment, however, this number is often limited by the nature (and density) of the network. The error made by the algorithm, when tracking 15 pixels of veins, is about $11^{\circ}$.

In a case study we applied our algorithms to the vein network of Physarum polycephalum (Fig. 1). The study concentrated only on the rear part of the network, which is less dense and more structured as in the apical zone.

The statistical analysis of data from a single experiment (or image) provided clear distributions of characteristic features of 

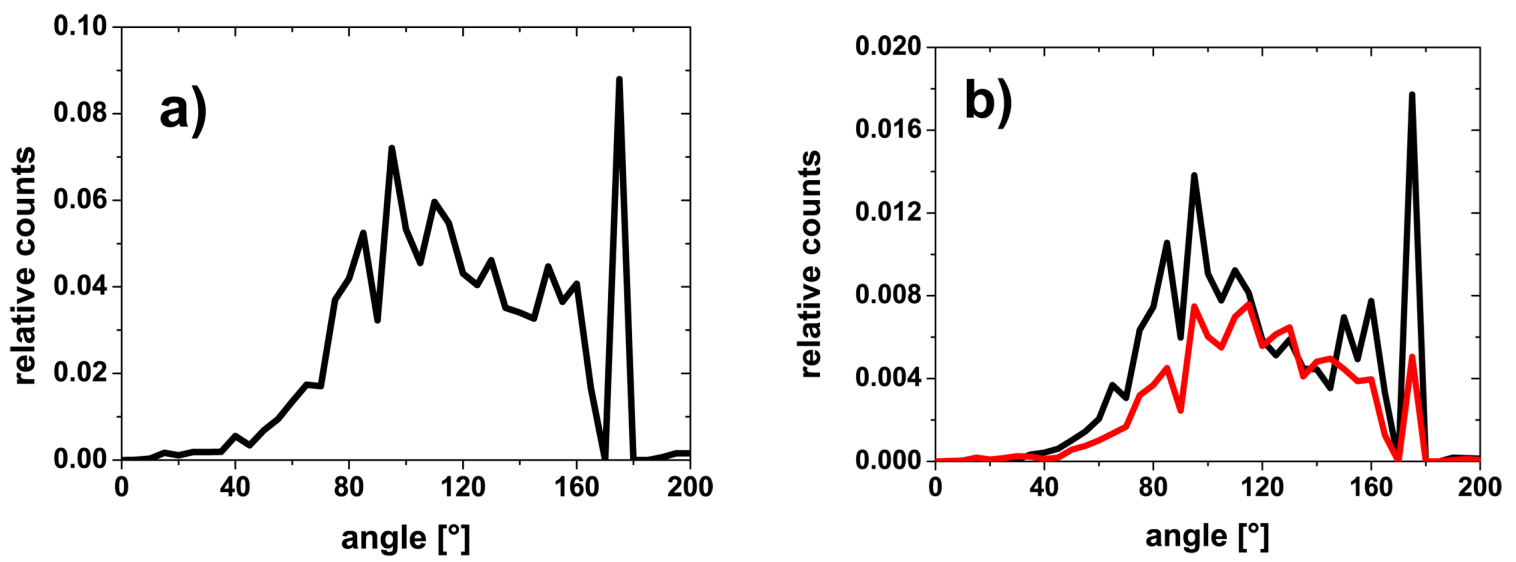

Figure 12 - a) Distribution of angles at branching points in the skeletonized network, based on 44000 angles. b) Distribution of angles at branching points in the skeletonized network in dependence of the relative thickness of the veins emanating from a node. The red line represents the case of strongly different thicknesses at branching points (i.e. $0.2 \leq \frac{w_{1}}{w_{3}} \leq 0.3$ ), while the black line represents veins with almost equal thicknesses at the branching points (i.e. $0.7 \leq \frac{w_{1}}{w_{3}} \leq 0.95$ ).

the veins (e.g. lengths, widths, etc.). However, due to the limited number of veins that can be collected in an image, large fluctuations are present in some of the statistics. Therefore, we also calculated the statistical distribution functions arising from $\mathrm{Cu}-$ mulative data of 121 independent experiments (as introduced in section 2.2.2). The same statistical distributions as those found in individual data can also be found in the cumulative data, thus showing that data from individual and cumulative images provide corresponding results. Obviously, however, the much bigger sample generated by cumulation of data leads to much smoother and less noisy statistical data.

In the case study of the behaviour of the $P$. polycephalum network, we have observed some interesting and intriguing behaviours. It has been found the mean length of the veins increases linearly with time while the number of veins forming the network decreases exponentially. While these two processes are due to the coarsening of the network, the physical reasons for the linear and exponential form of these correlations remain to be unravelled.

Bohn et al. [12] studied leaf venation networks. While the transport in leaf venation networks is passive, the network of P. polycephalum is actively pumping. Nevertheless, the distribution of thicknesses of both networks is log-normal. The distribution of angles at branching points of veins is similar when angles are not related to the relative widths of the veins at branching points. Both, the leaf and P. polycepha/um networks, have a continuous distribution with angles between $\sim 60^{\circ}$ and $180^{\circ}$. For leaf venation networks the distribution of angles changes drastically when relative widths of the veins are taken into account, while for $P$. polycephalum networks this behaviour is present, but barely detectable. We believe that the nature of the transport processes and the flow patterns generated by these processes may be at the origin of these differences.

\section{REFERENCES}

[1] BARABÁSI AL, JEONG H, NÉDA Z, RAVASZ E, SCHUBERT A \& VICSEK T. 2002. Evolution of the social network of scientific collaborations. Physica A, 311: 590-614.

[2] FAGIOLO G, REYES J \& SCHIAVO S. 2009. World-trade web: Topological properties, dynamics, and evolution. Phys. Rev. E, 79: 036115.

[3] BARRAT A, BARTHÉLEMY M, PASTOR-SATORRAS R \& VESPIGNANI A. 2004. The architecture of complex weighted networks. Proc. Natl. Acad. Sci., USA, 101: 3747-3752.

[4] GUIMERÀ R, MOSSA S, TURTSCHI A \& AMARAL LAN. 2005. The worldwide air transportation network: Anomalous centrality, community structure, and cities' global roles. Proc. Natl. Acad. Sci., USA, 102: 7794-7799.

[5] LI W \& CAI X. 2007. Empirical analysis of a scale-free railway network in China. Physica A, 382: 693-703.

[6] DOMÉNECH A. 2009. A topological phase transition between small-world and fractal scaling in urban railway transportation networks? Physica A, 388: 4658-4668.

[7] WATTS DJ \& STROGATZ SH. 1998. Collective dynamics of 'small world' networks. Nature, 393: 440-442.

[8] BUHL J, GAUTRAIS J, SOLÉ RV, KUNTZ P, VALVERDE S, DENEUBOURG JL \& THERAULAZ G. 2004. Efficiency and robustness in ant networks of galleries. Eur. Phys. J. B, 42: 123-129. 
[9] BUHL J, GAUTRAIS J, DENEUBOURG JL \& THERAULAZ G. 2004. Nest excavation in ants: group size effects on the size and structure of tunneling networks. Naturwiss., 91: 602-606.

[10] BUHL J, GAUTRAIS J, DENEUBOURG JL, KUNTZ P \& THERAULAZ G. 2006. The growth and form of tunneling networks in ants. J. Theor. Biol., 243: 287-298.

[11] GAZIT Y, BERK DA, LEUNIG M, BAXTER LT \& JAIN RK. 1995. Scale-invariant behaivor and vascular network formation in normal and tumor tissue. Phys. Rev. Lett., 75: 12, 2428.

[12] BOHN S, ANDREOTTI B, DOUADY S, MUNZINGER J \& COUDER Y. 2002. Constitutive property of the local organization of leaf venation networks. Phys. Rev. E, 65: 1-12.

[13] KATIFORI E, SZÖLLOSI GJ \& MAGNASCO MO. 2010. Damage and fluctuations induce loops in optimal transport networks. Phys. Rev. Lett., 104: 1-4.

[14] UEDA T. 2005. An inteligent slime mold: A self-organizing system of cell shape and information. In: ARMBRUSTER D, KANEKO $K$ \& MIKHAILOV AS (Eds.) Networks of interacting machines. World Scientific, New Jersey, pp. 221-255.

[15] BAUMGARTEN W, UEDA T \& HAUSER MJB. 2010. Plasmodial vein networks of the slime mold Physarum polycephalum form regular graphs. Phys. Rev. E, 82: 046113.
[16] TERO A, TAKAGI S, SAIGUSA T, ITO K, BEBBER DP, FRICKER MD, YUMIKI K, KOBAYASHI R \& NAKAGAKI T. 2010. Rules for biologically inspired adaptive network design. Science 327: 439-442.

[17] NAKAGAKI T, KOBAYASHI R, NISHIURA Y \& UEDA T. 2004. Obtaining multiple separated food sources: Behavioural intelligence in Physarum polycephalum. Proc. R. Soc. Lond. B, 271: 2305-2310.

[18] NAKAGAKI T, IIMA M, UEDA T, NISHIURA Y, SAIGUSA T, TERO A, KOBAYASHI R \& SHOWALTER K. 2007. Minimum-risk path finding by an adaptive amoebal network. Phys. Rev. Lett., 99: 068104.

[19] WATANABE S, TERO A, TAKAMATSU A \& NAKAGAKI T. 2011. Traffic optimization in railroad networks using an algorithm mimicking an amoeba-like organism, Physarum plasmodium. BioSystems, 105: 225-232.

[20] BAUMGARTEN W \& HAUSER MJB. 2010. Detection, extraction, and analysis of the vein network of the slime mould Physarum polycephalum. J. Comp. Interdisc. Sci., 1: 241-249.

[21] ZHANG T \& SUEN C. 1984. A fast parallel algorithm for thinning digital patterns. Comm. ACM, 27: 236-239.

[22] ITO M, OKAMOTO R \& TAKAMATSU A. 2011. Characterization of adaptation by morphology in a planar biological network of plasmodial slime mold. J. Phys. Soc. Japan, 80: 074801. 